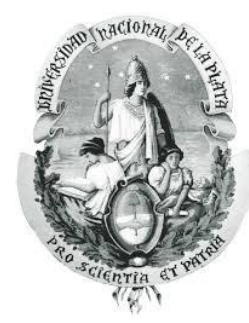

Maestría en Economía

Facultad de Ciencias Económicas

Universidad Nacional de La Plata

\title{
TESIS DE MAESTRÍA
}

ALUMNA

Ivana Benzaquén

TÍTULO

Efectos del Servicio de Atención Médica de Emergencias (SAME) sobre los fallecimientos por accidentes de tránsito en la provincia de Buenos Aires.

\section{DIRECTORA}

Mariana Marchionni

CO-DIRECTOR

Nicolás Epele

FECHA DE DEFENSA

Noviembre 2020

Códigos JEL: I10, R41 


\title{
Efectos del Servicio de Atención Médica de Emergencias (SAME) sobre los fallecimientos por accidentes de tránsito en la provincia de Buenos Aires.
}

\author{
Ivana Benzaquén ${ }^{1}$
}

\section{Resumen}

Este trabajo evalúa el impacto de la implementación del Servicio de Atención Médica de Emergencias (SAME) en la provincia de Buenos Aires sobre las muertes por accidentes viales. A partir de información mensual de accidentes de tránsito de los 135 municipios de la provincia para los años 2016, 2017 y 2018, se explota la variabilidad en el momento de implementación del SAME entre los municipios y el hecho de que algunos nunca adoptaron el servicio para identificar el efecto promedio de la intervención en los municipios con SAME mediante la metodología de Diferencias en Diferencias. El principal resultado es que la incorporación del SAME produjo una reducción promedio del 19\% en la tasa de defunciones cada mil accidentados con respecto a su línea de base. Además, se realizan múltiples chequeos de robustez y los resultados se mantienen. Se concluye que sería deseable expandir el SAME a otros municipios y/o provincias.

Palabras clave: servicio de emergencias, SAME, defunciones por accidentes de tránsito, seguridad vial, diferencias en diferencias, evaluación de impacto.

Clasificación JEL: I10, R41

\footnotetext{
${ }^{1}$ El presente trabajo constituye mi tesis de Maestría en Economía de la Facultad de Ciencias Económicas de la Universidad Nacional de La Plata, realizada bajo la dirección de Mariana Marchionni y Nicolás Epele, a quienes agradezco enormemente por toda la orientación y el apoyo que me brindaron a lo largo de este proceso. También agradezco los excelentes comentarios de Guillermo Falcone, Mariana Conte Grand, Florencia Pinto, Natalia Porto, Inés Berniell y de los participantes del seminario de avance de tesis. Por último, agradezco a Camila Cabandié por la generosidad de compartirme información sobre el funcionamiento del SAME. Del desarrollo de esta investigación se desprendieron los resultados presentados en el informe de gestión sobre evaluación de impacto de políticas provinciales del Ministerio de Economía de la Provincia de Buenos Aires. Cualquier error u omisión es de mi exclusiva responsabilidad. Correo electrónico: ivanabenzaquen@ gmail.com.
} 


\section{Introducción}

En el año 2015, la Organización de las Naciones Unidas aprobó la Agenda 2030 sobre el Desarrollo Sostenible, dentro de la cual se ha fijado una meta ambiciosa con respecto a la seguridad vial que consiste en reducir a la mitad el número de defunciones y lesiones por accidentes de tránsito en todo el mundo para el año $2020 .^{2}$

Los accidentes viales representan uno de los principales problemas de la salud pública: cada año mueren cerca de 1.3 millones de personas en las carreteras del mundo entero, y entre 20 y 50 millones padecen traumatismos no mortales. Se trata de una de las principales causas de muerte en todos los grupos etarios, y la primera entre personas de 15 a 29 años. A su vez, más de la mitad de las defunciones afectan a usuarios vulnerables de la vía pública, es decir, peatones, ciclistas y motociclistas (WHO, 2018).

Por otra parte, las lesiones causadas por accidentes de tránsito ocasionan pérdidas económicas considerables para las personas, sus familias y los países en su conjunto, que son consecuencia de los costos de tratamiento, de la pérdida de productividad de las víctimas y/o de sus familiares, de los costos administrativos y de daños a la propiedad, y de la desutilidad que las lesiones o la pérdida de vida conlleva. Por ejemplo, para la mayor parte de los países los accidentes viales representan un costo de entre el 1\% y el 3\% de su PIB, y además el 90\% de las defunciones y traumatismos causados por accidentes de tránsito se producen en países de ingresos bajos y medios (WHO, 2015; 2017; 2018).

Con el objetivo de reducir las cifras previstas de víctimas mortales en accidentes de tránsito en todo el mundo, el Grupo de Colaboración de las Naciones Unidas para la Seguridad Vial elaboró el Plan Mundial para el Decenio de Acción para la Seguridad Vialª que proporciona un marco general de acción, promoviendo soluciones económicas y de eficacia probada para mejorar la seguridad vial en las siguientes esferas: (1) gestión de la seguridad vial, (2) vías

\footnotetext{
${ }^{2}$ Se trata de la Meta 3.6 dentro del Objetivo 3: "Garantizar una vida sana y promover el bienestar para todos en todas las edades."

${ }^{3}$ En el año 2010 la Asamblea General de las Naciones Unidas adoptó la resolución 64/255, que proclamaba el período 2011-2020 como el Decenio de Acción para la Seguridad Vial.
} 
de tránsito y movilidad más seguras, (3) vehículos más seguros, (4) usuarios de vías de transito más seguros y (5) respuesta tras los accidentes.

Idealmente, los países deberían hacer un esfuerzo integral sin descuidar ninguno de los pilares mencionados, para poder lograr la construcción de un sistema vial seguro que abarque desde la prevención de los accidentes hasta la atención oportuna de las víctimas. Sin desconocer la perspectiva global con la que debe tratarse este problema, en el presente trabajo se resaltará la importancia del quinto pilar: brindar una respuesta adecuada luego de un accidente.

La atención médica tras un accidente vial tiene plazos críticos. Cualquier demora para detectar lesiones y prestar asistencia a las víctimas aumenta la gravedad de los traumatismos, e incluso en ciertos casos, tan solo unos minutos de retraso pueden suponer la diferencia entre la vida y la muerte. Es por ello que el acceso a una atención de emergencia eficaz y oportuna salva vidas y reduce el riesgo de discapacidad entre los lesionados (WHO, 2017; Clark et al., 2012; Clark et al., 2013; Travis et al., 2012; Vasudevan et al., 2016).

Entre las soluciones clave para establecer un sistema de atención de emergencia se destacan: el establecimiento de números telefónicos de acceso universales vinculados a servicios integrados de asistencia pre hospitalaria de emergencia en los centros de salud, la capacitación de los profesionales sanitarios de primera línea en atención básica de emergencia, y la formación de los primeros intervinientes no profesionales en los lugares donde los sistemas formales sean limitados (WHO, 2015; 2017; 2018).

En la provincia de Buenos Aires (PBA), que reúne casi el 40\% de la población de la Argentina, cada año mueren alrededor de 1300 personas debido a accidentes de tránsito, mientras que unas 40 mil sufren algún tipo de lesión a causa de estos incidentes. Teniendo en cuenta que la PBA tiene aproximadamente 17 millones de habitantes, estas cifras representan cerca de 8 muertes y 235 accidentados cada 100 mil habitantes por año. A su vez, la mayor parte de las defunciones se da entre jóvenes de 15 a 29 años, hombres, motociclistas y peatones (DPE, 2019). ${ }^{4} \mathrm{Si}$ se compara con cifras nacionales, según

\footnotetext{
${ }^{4}$ En el anexo se presentan estadísticas descriptivas de los fallecimientos por accidentes viales en la PBA.
} 
estadísticas de la Agencia Nacional de Seguridad Vial (2019), alrededor del 25\% de las muertes por accidentes viales en todo el país se producen en la PBA.

En este contexto, a fines de 2016 comenzó a implementarse el Servicio de Atención Médica de Emergencias (SAME) en la PBA. El SAME es un servicio de atención ambulatorio gratuito con un número telefónico único (107) para todo el territorio provincial, que se incorporó de manera secuencial en los distintos municipios de la PBA. Hacia fines de 2018, 87 de los 135 municipios de la provincia ya contaban con este servicio. ${ }^{5}$

A la luz de lo expuesto, el objetivo de este trabajo es evaluar si la implementación del SAME contribuyó a reducir las muertes por accidentes viales en la PBA en el período 2016-2018. Para ello se explota la variabilidad en el momento de implementación del SAME entre los municipios y el hecho de que algunos nunca adoptaron el servicio para identificar el efecto promedio de los municipios con SAME mediante la metodología de Diferencias en Diferencias.

Los resultados sugieren una disminución promedio del 19\% de la tasa de defunciones cada mil accidentados con respecto a su línea de base. Estos resultados son robustos a distintas especificaciones. Adicionalmente, se realizó una descomposición del efecto estimado para explorar posibles heterogeneidades como consecuencia de la secuencialidad con que el SAME fue adoptado en los municipios. Para ello se utilizó la metodología propuesta recientemente por Goodman-Bacon (2018) y se encontró que el 44\% del efecto encontrado surge de la variabilidad en los momentos de implementación del SAME entre municipios.

El resto del trabajo se organiza de la siguiente manera: en la sección 2 se realiza una revisión de la literatura; luego se describen las principales características del SAME en la sección 3 y se presentan los datos y la metodología en la sección 4. La sección 5 muestra los resultados, y en la 6 se realizan controles de robustez. Finalmente, en la sección 7 se exponen las conclusiones del trabajo.

\footnotetext{
${ }^{5}$ Hasta la fecha son más de 100 los municipios que implementaron el SAME; pero dada la disponibilidad de información este trabajo se circunscribe al período 2016-2018.
} 


\section{Antecedentes}

Varios artículos en la literatura estudian la relación entre el resultado de un accidente y la provisión de atención médica. Desde una perspectiva agregada, Noland (2003) y Noland y Quddus (2004) señalan que las mejoras de la atención médica y la tecnología son factores que contribuyen a la reducción de muertes por accidentes de tránsito en los países industrializados. En este sentido, el trabajo de Castillo-Manzano et al. (2014) con datos de 27 países de la Unión Europea del período 1999-2009 encuentra que la densidad de camas hospitalarias contribuye a una disminución de las defunciones relacionadas con el tráfico. Además, obtiene que la calidad de las instalaciones médicas generales y la tecnología asociada con aumentos en el gasto público en salud también puede ser un factor relevante para reducir este tipo de muertes.

A nivel micro, la mayoría de los artículos que analizan este problema se han centrado en dos factores que pueden ayudar a reducir los fallecimientos por accidentes de tránsito. Por un lado, una notificación rápida y precisa a los servicios de emergencia (Brodsky, 1990, 1992, 1993; Evanco, 1999; Clark y Cushing, 2002) y por otro, la distancia desde el lugar del accidente hasta un centro médico (Bentham, 1986; Muelleman y Mueller, 1996; Durkin et al., 2005; Zwerling et al., 2005; Li et al., 2008). Por ejemplo, Murad et al. (2012) en un estudio para Irak encuentran que en comunidades de bajos recursos con largos tiempos de transporte ambulatorio, un sistema prehospitalario de paramédicos capacitados reduce significativamente la mortalidad por trauma en las lesiones graves de accidentes de tránsito.

Otro factor importante para reducir las defunciones por siniestros viales es el tiempo de llegada de la ambulancia a la escena del accidente. En este sentido, el trabajo de SanchezMangas et al. (2010) utiliza una muestra de accidentes ocurridos en carreteras españolas en mayo de 2004, y encuentra evidencia de que una reducción en 10 minutos de llegada del servicio de emergencia se asocia con una disminución promedio de la probabilidad de muerte en un tercio, tanto en autopistas como en carreteras convencionales. En la misma línea, Clark et al. (2013), en un estudio para Estados Unidos, encuentran evidencia que apoya la intuición de lo que se conoce como la "hora dorada" en la atención de servicios de emergencia y la importancia del transporte oportuno a un hospital. 
Finalmente, en el trabajo de Vasudevan et al. (2016) se realiza un análisis a partir de la experiencia de los servicios de emergencia en India, y encuentran que, a pesar de tener un sistema eficiente, no se logra una mejora significativa en la seguridad vial debido a que en general las personas desconocen la existencia de estos servicios. La mayoría de la población opta por llevar a la víctima al hospital utilizando sus vehículos personales o cualquier otro vehículo disponible, en lugar de llamar a una ambulancia.

\section{El Servicio de Atención Médica de Emergencias (SAME)}

\section{Funcionamiento del SAME}

El SAME es un servicio integral de emergencias y cobertura ante accidentes, que comenzó a funcionar en septiembre de 2016 en la provincia de Buenos Aires, Argentina. Es de amplia cobertura territorial y brinda una respuesta rápida, cotidiana y con personal especializado. El servicio está organizado en bases distribuidas geográficamente de manera estratégica para poder asistir eficazmente ante situaciones de emergencias médicas. El SAME ofrece asistencia las 24 horas y funciona en forma coordinada con los establecimientos dedicados a la atención de la salud radicados en los municipios (hospitales, clínicas, salas sanitarias, etc.). Es decir, su acción complementa la de los diferentes actores con competencia en materia de urgencias y emergencias para fortalecer las prácticas de atención primaria y emergencias. Además, el SAME ofrece cobertura gratuita para todas las personas, sin distinción de edad y tengan o no seguro de salud, ante accidentes en la vía pública o durante situaciones de emergencia.

Operativamente el SAME adopta un protocolo para medicina de emergencias y desastres avalado internacionalmente, conocido como triage. Se trata de un procedimiento de selección y clasificación de pacientes que evalúa las prioridades de atención, privilegiando la posibilidad de supervivencia de acuerdo con las necesidades terapéuticas y los recursos disponibles. El objetivo es evitar que se retrase la atención de aquel paciente cuyo pronóstico 
empeoraría por la demora en la atención. A tal efecto, se establecen cuatro niveles de prioridad, descriptos en la Tabla $1 .^{6}$

Tabla 1. Clasificación de los niveles de prioridad del triage. ${ }^{7}$

\begin{tabular}{ll}
\hline Código & \multicolumn{1}{c}{ Significado } \\
Rojo & $\begin{array}{l}\text { Emergencia: urgencia vital, riesgo de vida } \\
\text { inminente. }\end{array}$ \\
Amarillo & $\begin{array}{l}\text { Urgencia: urgencia no vital, sin riesgo de vida } \\
\text { inminente, pero la atención no debe demorarse. } \\
\text { Consulta Médica: urgencia no vital, no hay riesgo } \\
\text { Verde vida. }\end{array}$ \\
Negro & $\begin{array}{l}\text { Óbito. Se debe dar intervención policial. } \\
\text { Fuente: Manual de Atención de Emergencias Médicas de SAME Provincia de Buenos Aires }\end{array}$
\end{tabular}

El SAME funciona siguiendo un detallado protocolo en el que se identifican las actividades principales de cada participante, se establecen códigos y se detalla una secuencia ordenada y mecánica de pasos a seguir ante cada posible escenario.

Primero, los operadores de radio reciben y canalizan las demandas por situaciones de emergencia, realizando un breve interrogatorio para obtener la ubicación exacta y las características del hecho y de las víctimas. En base a la información recabada, el radio operador utiliza el triage para clasificar la emergencia y se lo comunica al equipo médico, que se dirige en ambulancia al lugar del hecho. Una vez allí, el médico a cargo evalúa la escena para identificar posibles riesgos para el personal y determina si es necesario solicitar otras unidades de ayuda.

A continuación, el médico procede a la evaluación de la víctima: examina su estado de consciencia, verifica respiración y ventilación, estado circulatorio y control de hemorragias, determina si hay déficit neurológico, resguarda de hipotermia y toma signos vitales. En base

\footnotetext{
${ }^{6}$ El término triage proviene del francés y significa "separar, clasificar". Comenzó a utilizarse como un sistema de clasificación para tratar a los heridos en el campo de batalla durante las guerras napoleónicas. Desde entonces el triage fue utilizado en medicina militar, y con el correr del tiempo se adaptó a contextos civiles, particularmente en medicina de emergencias y desastres, siendo hoy una práctica extendida y reconocida en todo el mundo (Iserson y Moskop, 2007).

${ }^{7}$ En la tabla B.1 del anexo B se muestran algunos ejemplos de situaciones de emergencias clasificados según los códigos del triage.
} 
a la evaluación recategoriza la emergencia, informando al conductor de la ambulancia para que comunique a la central de despacho el cambio de triage para su registro.

Luego de la evaluación, el médico activa la cadena de supervivencia mediante acciones como la reanimación cardiopulmonar y la aplicación de desfibrilador externo automático, ofreciendo apoyo vital y traslado del paciente. En caso de haber víctimas múltiples o en masa, se sigue el protocolo del triage.

En la etapa de traslado, el médico comunica el nivel de complejidad requerido para el tratamiento de la víctima a la central de despacho y ésta determina cuál es el centro de salud que mejor se adecúa a las características de la emergencia y a la ubicación. Finalmente, el paciente se entrega personalmente al médico responsable notificado por la central y se describe su situación, el tratamiento administrado y su evolución. ${ }^{8}$

\section{Implementación del SAME}

La decisión de implementar el servicio es de los municipios. Cada municipio adherente debe contar con bases fijas, radio-operadoras, corredores sanitarios nominalizados y protegidos, recursos humanos altamente capacitados (médicos, conductores de ambulancias, coordinadores médicos, telefonistas, radioperadores, administrativos y capacitadores), y recursos materiales de calidad (móviles de emergencia, instrumental médico, indumentaria, combustible, comunicación y mecanismos de coordinación). Si bien esta autoselección en la adopción del servicio constituye una potencial amenaza para la identificación de su efecto, veremos más adelante que no hay evidencia de selección basada en características asociadas a la tasa de defunciones en accidentes de tránsito.

De los 135 municipios bonaerenses, en total son 87 los que implementaron el SAME en el período 2016-2018. La incorporación secuencial se ilustra en la Tabla 2.

\footnotetext{
${ }^{8}$ En el anexo B se detalla el equipamiento de las ambulancias.
} 
Tabla 2. Cronograma de implementación del SAME.

\begin{tabular}{lccc}
\hline Mes & $\begin{array}{c}\text { Cantidad } \\
\text { acumulada de } \\
\text { municipios } \\
\text { con SAME }\end{array}$ & $\begin{array}{c}\text { Porcentaje } \\
\text { acumulado de } \\
\text { municipios } \\
\text { con SAME }\end{array}$ & $\begin{array}{c}\text { Porcentaje } \\
\text { acumulado de } \\
\text { población } \\
\text { cubierta por } \\
\text { SAME }\end{array}$ \\
\hline sep-16 & 2 & $1 \%$ & $5 \%$ \\
dic-16 & 3 & $2 \%$ & $8 \%$ \\
ene-17 & 6 & $4 \%$ & $14 \%$ \\
mar-17 & 14 & $10 \%$ & $26 \%$ \\
abr-17 & 17 & $13 \%$ & $37 \%$ \\
may-17 & 23 & $17 \%$ & $40 \%$ \\
jun-17 & 24 & $18 \%$ & $40 \%$ \\
jul-17 & 28 & $21 \%$ & $42 \%$ \\
ago-17 & 31 & $23 \%$ & $44 \%$ \\
sep-17 & 34 & $25 \%$ & $46 \%$ \\
oct-17 & 35 & $26 \%$ & $48 \%$ \\
nov-17 & 37 & $27 \%$ & $48 \%$ \\
ene-18 & 40 & $30 \%$ & $49 \%$ \\
feb-18 & 43 & $32 \%$ & $50 \%$ \\
mar-18 & 46 & $34 \%$ & $50 \%$ \\
abr-18 & 48 & $36 \%$ & $50 \%$ \\
may-18 & 52 & $39 \%$ & $51 \%$ \\
jun-18 & 57 & $42 \%$ & $52 \%$ \\
jul-18 & 61 & $45 \%$ & $55 \%$ \\
ago-18 & 68 & $50 \%$ & $56 \%$ \\
sep-18 & 75 & $56 \%$ & $57 \%$ \\
oct-18 & 80 & $59 \%$ & $58 \%$ \\
nov-18 & 84 & $62 \%$ & $61 \%$ \\
dic-18 & 87 & $64 \%$ & $68 \%$ \\
\hline & & & \\
\hline
\end{tabular}

Fuente: elaboración propia en base a información provista por el Ministerio de Salud de la PBA.

Los dos primeros municipios en implementar el SAME lo hicieron en septiembre de 2016. Un año más tarde, el 25\% de los municipios de la provincia (34 municipios) contaba con el servicio, cubriendo a su vez al $46 \%$ de la población bonaerense. La implementación siguió aumentando hasta llegar a fines de 2018 a un 64\% de municipios con SAME y 68\% de población cubierta. La Figura 1 ilustra la distribución geográfica de los municipios y los meses transcurridos hasta diciembre de 2018 desde la implementación del SAME. 
Figura 1. Meses transcurridos desde la implementación del SAME en los municipios de la provincia de Buenos Aires.

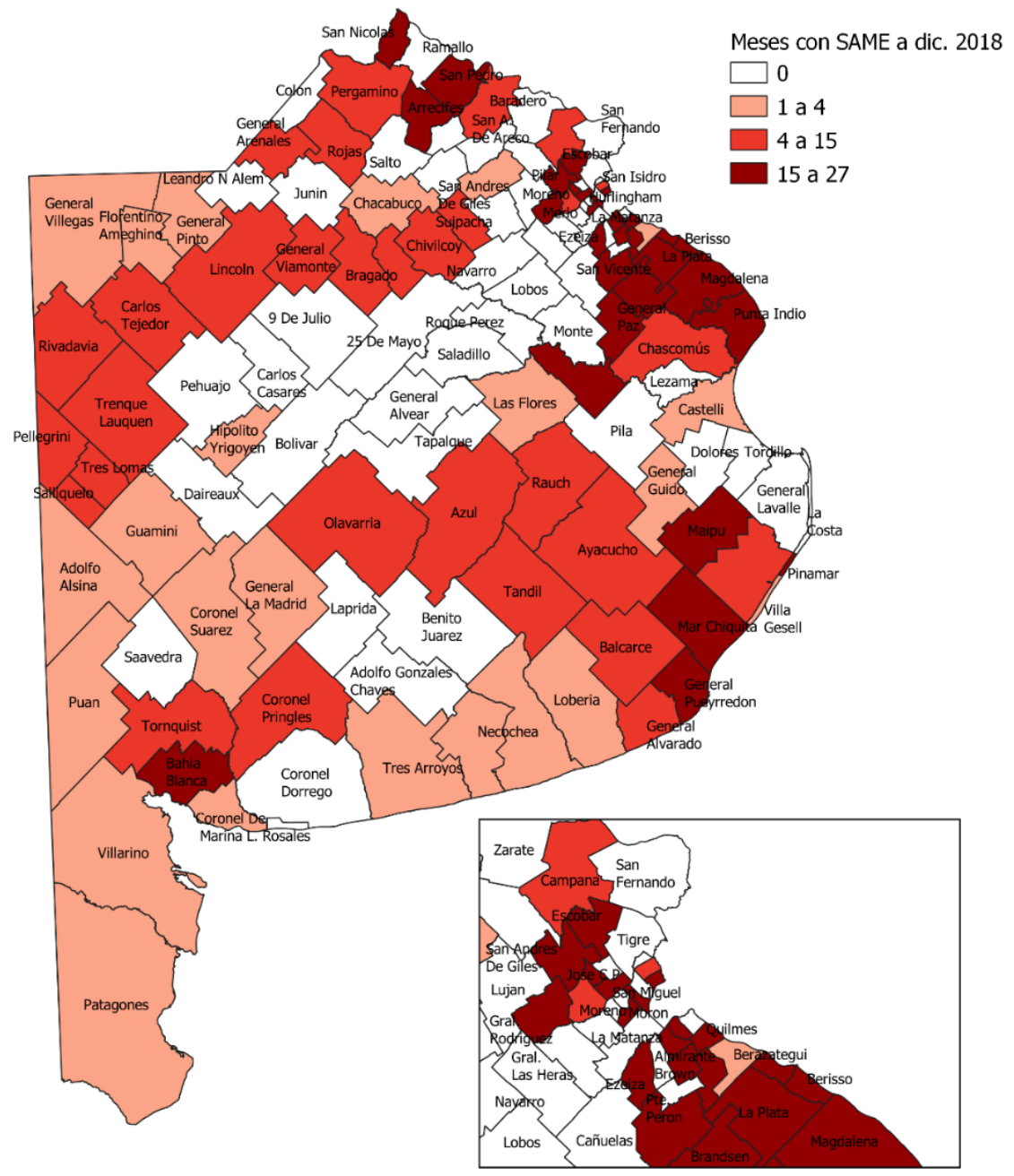

Fuente: elaboración propia en base a información provista por el Ministerio de Salud de la PBA.

Como se mencionó antes, la autoselección de los municipios en la adopción del SAME constituye una potencial amenaza para identificar su impacto. Por ejemplo, en el período considerado el gobierno provincial llevó adelante obras de reconstrucción, rehabilitación y repavimentación de rutas provinciales. ${ }^{9}$ Es razonable suponer que la inversión en infraestructura vial afecte el número de siniestros viales y por ende la mortalidad asociada a dicha causa. Por lo tanto, una preocupación importante para la estrategia de identificación es que la inversión en obras viales pueda estar correlacionada con la decisión de implementar

\footnotetext{
${ }^{9}$ La mayoría de estas obras comenzaron en el año 2017 y muchas de ellas todavía están en ejecución.
} 
el SAME. En principio no habría razones para creer que estas dos políticas estén relacionadas, dado que la inversión en obras viales es llevada a cabo por el gobierno provincial, mientras que la implementación del SAME es una decisión de cada municipio. Pero para ganar confianza se analizaron los patrones de implementación del SAME en función de distintas características de los municipios, entre ellas si el partido fue afectado por las obras viales.

La Tabla 3 muestra los resultados de estimar por mínimos cuadrados ordinarios una regresión de la probabilidad de tener SAME (columna 1) y el número de meses con SAME (columna 2) en función de distintas características de los municipios: si el gobierno municipal es del mismo partido político que el gobierno provincial en 2016 (Oficialista vale 1 si hay coincidencia del partido político y 0 en caso contrario), un indicador que identifica los municipios del área metropolitana de la ciudad de Buenos Aires (Conurbano = 1), el porcentaje de hogares con al menos una NBI en 2010, el porcentaje de población con obra social o prepaga en 2010, un indicador de si el municipio fue afectado por obras viales en rutas durante el período de análisis, la tasa de defunciones cada mil accidentados en 2016, la tasa de accidentes cada 100 mil habitantes en 2016, la población municipal de 2016 en logaritmos y la superficie del municipio. ${ }^{10}$

\footnotetext{
${ }^{10}$ En el Anexo C se presenta la definición de todas las variables y sus respectivas fuentes.
} 
Tabla 3. Estimación de la probabilidad de adhesión al SAME.

\begin{tabular}{lll}
\hline & $\begin{array}{l}\text { Implementó } \\
\text { SAME }\end{array}$ & $\begin{array}{l}\text { Cantidad de } \\
\text { meses con } \\
\text { SAME }\end{array}$ \\
\hline Oficialista & $0.454^{* * *}$ & $6.458^{* * *}$ \\
& $(0.0868)$ & $(1.472)$ \\
Conurbano & 0.0988 & -0.478 \\
& $(0.191)$ & $(3.238)$ \\
\% hogares con al menos una NBI & 0.0305 & 0.194 \\
\% población con obra social/prepaga & $(0.0254)$ & $(0.431)$ \\
& 0.00887 & 0.0465 \\
Obras viales en rutas & $(0.0105)$ & $(0.178)$ \\
& -0.0883 & 0.677 \\
Tasa de defunciones cada mil accidentados & $(0.112)$ & $(1.903)$ \\
Tasa de accidentados cada 100 mil habitantes & -0.000588 & -0.000103 \\
& $(0.000452)$ & $(0.00766)$ \\
Población $(\log )$ & 0.00134 & 0.0232 \\
& $(0.00186)$ & $(0.0315)$ \\
Superficie $\left(\mathrm{km}^{2}\right)$ & 0.0202 & $1.971 * *$ \\
& $(0.0476)$ & $(0.807)$ \\
Constante & $2.46 \mathrm{e}-05$ & $-0.000774^{*}$ \\
& $(2.52 \mathrm{e}-05)$ & $(0.000428)$ \\
\hline Observaciones & -0.531 & -20.53 \\
R2 & $(1.163)$ & $(19.71)$ \\
F-obs & 134 & 134 \\
Not & 0.460 & 0.466 \\
\hline
\end{tabular}

Nota: Los errores estándar se presentan entre paréntesis. Todas las regresiones incluyen efectos fijos por región sanitaria y por zona vial. *** $\mathrm{p}<0.01, * * \mathrm{p}<0.05, * \mathrm{p}<0.10$

Los resultados de la Tabla 3 sugieren que la decisión de implementación del SAME por parte de los municipios no está asociada ni con la tasa de defunciones ni con otras características que puedan afectarla. En particular, la única variable significativa para explicar la probabilidad de adoptar el SAME es Oficialista, lo que posiblemente se deba a las facilidades políticas de gestionar un convenio cuando el gobierno municipal es del mismo partido político que el gobierno provincial.

En la segunda columna se refuerza este hallazgo, encontrando que además de tener mayor probabilidad de adhesión, los municipios oficialistas son los primeros en incorporar el servicio. Sin embargo, no hay razones para sospechar que el signo del partido político tenga alguna relación con la tasa de defunciones por accidentes. De la segunda columna también surge que en promedio fueron los municipios con mayor población y menor tamaño (es decir, 
con mayor densidad poblacional) los primeros en implementar el SAME. El análisis propuesto en la siguiente sección controla por estas características prácticamente invariantes durante el periodo de análisis mediante efectos fijos por municipio.

\section{Datos y metodología}

La Organización Mundial de la Salud (OMS) considera que los fallecimientos en incidentes viales son aquellas muertes producidas como consecuencia de atropellos y colisiones de tránsito, independientemente del tiempo transcurrido desde el accidente (WHO, 2015). Asimismo, el trabajo de Jacobs et al. (2000) documenta que la mayoría de los fallecimientos por accidentes de tránsito suceden dentro de los 30 días del hecho. Esto proporciona un marco para definir a los fallecidos por accidentes viales como aquellas muertes por atropellos y colisiones de tránsito ocurridas dentro de los 30 días del incidente. ${ }^{11}$

Los principales organismos que disponen de fuentes de información y registro sobre accidentes de tránsito en la PBA son: 1) Ministerio de Seguridad, que brinda la información referente a los lesionados y fallecidos en el lugar del hecho; 2) Ministerio de Salud, que dispone la información para darle seguimiento a los lesionados y fallecidos dentro de los 30 días post siniestro a través de las bases de egresos hospitalarios y las defunciones generales y 3) el Ministerio Público de la provincia de Buenos Aires que, a través de la información de las causas iniciadas por homicidios culposos, corrobora el motivo de fallecimiento en caso de defunciones con causas externas sin especificar y complementa la información faltante en una u otra base.

A partir del año 2016, el Observatorio Vial de la Provincia de Buenos Aires en conjunto con la Dirección Provincial de Estadística de la Provincia de Buenos Aires (DPE) realizan un minucioso trabajo donde recopilan y unifican los datos de las fuentes mencionadas, consolidándolos en una única base de datos homogénea y confiable. De esta manera, el análisis cuantitativo se hará en base a información mensual para cada municipio provista por la DPE y el Observatorio Vial de la PBA en el período 2016-2018, sobre la cantidad de

\footnotetext{
${ }^{11}$ Cabe aclarar que si bien se podría prolongar el período luego de los 30 días, eso implicaría solo un aumento marginal en las cifras de fallecidos, mientras que los esfuerzos de recolección de datos serían desproporcionadamente grandes.
} 
fallecidos y lesionados en accidentes de tránsito. A su vez se cuenta con la fecha de implementación del SAME para cada municipio provista por el Ministerio de Salud de la Provincia de Buenos Aires. De esta manera se tiene un panel balanceado de 4860 observaciones (135 municipios con información de 36 meses), y la variable de interés será la cantidad de fallecidos cada mil accidentados por siniestros viales por mes. A su vez, en este trabajo se define como accidentados a la suma entre lesionados y fallecidos por accidentes de tránsito. ${ }^{12}$

En cuanto a la metodología, se utiliza el método de Diferencias en Diferencias (Card, 1990; Card y Krueger, 1994) para estimar el efecto promedio de la implementación del SAME sobre las defunciones por accidentes viales. En nuestro caso, el método de Diferencias en Diferencias permite explotar el hecho de que algunos municipios nunca implementaron el SAME y otros sí, y que dentro del último grupo hay variabilidad en el momento de implementación. Formalmente, el método de diferencias en diferencias se puede representar como un modelo de regresión lineal con efectos fijos por partido:

$$
\text { tasa_defunciones }_{i t}=\alpha+\beta \text { same }_{i t}+\delta X_{q i}+\lambda_{t}+\mu_{i}+\varepsilon_{i t}
$$

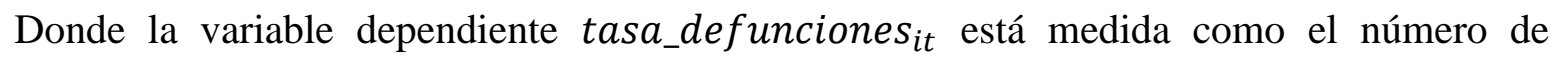
defunciones cada mil accidentados del municipio $i$ en el mes $t$; same $e_{i t}$ es una variable binaria que vale 1 si el municipio i tiene SAME en el mes $t$ y 0 en caso contrario, $X_{q i}$ es la interacción entre un vector de características del municipio $i$ (tasa de accidentados, región sanitaria, zona vial y color político) y dummies de trimestre $q^{13}, \mu_{i}$ es un efecto fijo del municipio $i, \lambda_{t}$ es un efecto del mes $t$ y $\varepsilon_{i t}$ es el término de error idiosincrático que varía entre municipios y en el tiempo, y que se supone distribuido independientemente de $\mu_{i}$ y $\lambda_{t}$. En este modelo, $\beta$ es el estimador de Diferencias en Diferencias del impacto promedio de la implementación del SAME en la tasa de defunciones viales, es decir, el efecto promedio del tratamiento sobre los tratados.

\footnotetext{
${ }^{12}$ Cabe aclarar que dentro de los lesionados se encuentran las personas involucradas en aquellos accidentes de tránsito en los que acudió la policía. Es decir, colisiones menores que se resuelven de forma privada no están registradas en esta base de datos.

${ }^{13}$ Debido a que las características por municipio no tienen variabilidad temporal, para poder incluirlas en el modelo de efectos fijos se las interactúa con dummies trimestrales (el panel contiene 12 trimestres).
} 
Los supuestos clave para la identificación del impacto causal de la implementación del SAME son que en su ausencia, la tendencia de la tasa de defunciones del grupo de tratamiento hubiera sido igual a la del grupo de control, y que no hubo otro evento diferente a la implementación del SAME que implique un impacto diferencial entre grupos sobre las defunciones por accidentes viales. En este sentido, las obras viales en rutas que se llevaron a cabo en este periodo podrían constituir una amenaza a la identificación del efecto del SAME, pero como se mostró en la sección anterior no hay evidencia de que estas obras se hayan concentrado en los municipios que implementaron el servicio de emergencias ni en los que lo implementaron más tempranamente.

\section{Resultados}

La Figura 2 presenta evidencia preliminar del efecto del SAME sobre la tasa de defunciones viales. En la figura se compara la evolución de la tasa de defunciones de los partidos que adoptaron el SAME versus los que no lo hicieron. Mediante las barras se representa el porcentaje de municipios con SAME por mes.

Figura 2. Evolución de la tasa de defunciones cada mil accidentados para municipios con SAME vs. sin SAME. Media móvil de 4 meses.

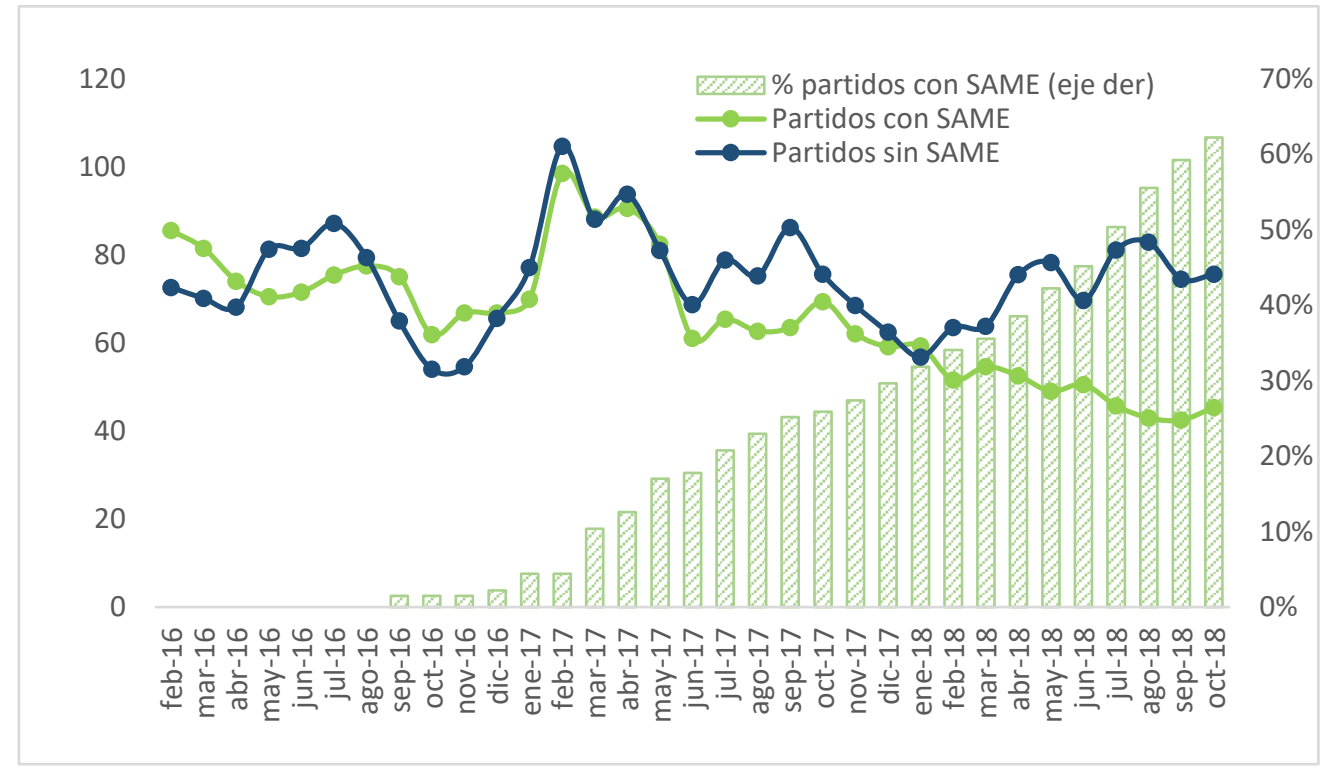

Fuente: elaboración propia en base a información provista por el Ministerio de Salud de la PBA. 
Se observa que tanto el nivel como la tendencia de la tasa de defunciones es similar hasta mediados de 2017, momento en el que la proporción de partidos con SAME era aproximadamente de $20 \%$. A partir de entonces las tendencias comienzan a divergir (con excepción de un breve periodo hacia fines de 2017) y el hecho de que la brecha sea cada vez más grande es consistente con la implementación secuencial de este servicio a lo largo de los municipios y con un efecto negativo del SAME sobre la tasa de defunciones.

La evidencia de la Figura 3 apunta en la misma dirección. Aquí se muestra la cantidad de defunciones (eje izquierdo) y de lesionados (eje derecho) con relación a una variable de tiempo relativo al mes de implementación del SAME. Es decir, para cada municipio el valor cero en el eje horizontal corresponde al mes en que se implementó el SAME, de manera que valores negativos (positivos) de esa variable indican la cantidad de meses previos (posteriores) a la implementación.

Se observa que, a partir de la implementación del SAME (barra roja), las defunciones disminuyen, al tiempo que los lesionados aumentan. Este resultado tiene sentido y es consistente con un efecto negativo del SAME en la tasa de muertes por accidentes: suponiendo que la cantidad total de accidentados es exógena, una reducción de los fallecidos debería corresponderse con un aumento de los lesionados por accidentes viales. 
Figura 3. Evolución de las defunciones y lesionados en partidos con SAME alrededor del mes de su implementación.

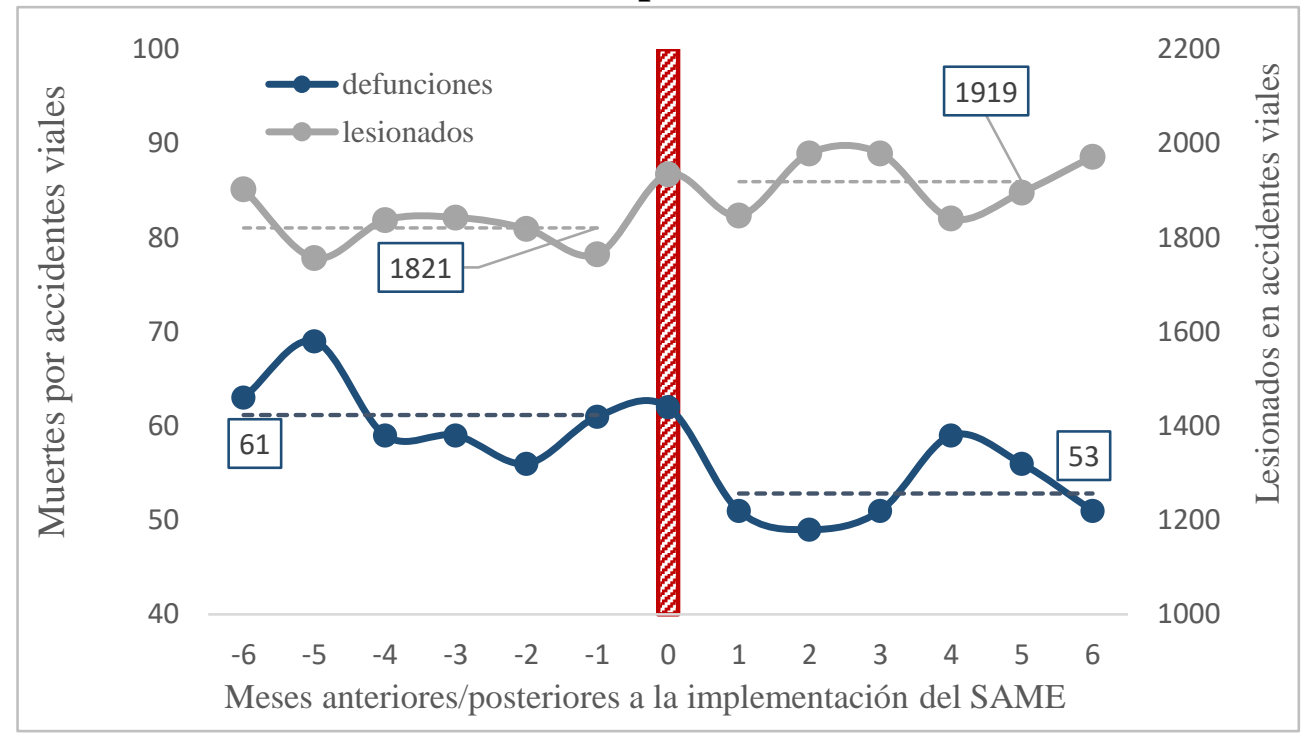

Nota: las líneas punteadas indican el promedio de cada variable antes y después de la implementación del SAME.

Naturalmente la variable temporal está definida sólo para aquellos municipios que hayan implementado el servicio. A su vez se elige una ventana de tiempo $t \in(-\mathrm{T}, \mathrm{T})$ para contar con un soporte común de municipios, debido a que el rango que toma $t$ para cada municipio es distinto. Más específicamente, de los 87 municipios que implementaron SAME en el período relevante, se realiza una submuestra de 47 en el rango $t \in(-6,6)$, es decir 6 meses antes y 6 meses después de la implementación. En el Anexo D se presentan dos variantes de esta figura con ventanas de tiempo de 3 y 4 meses.

La Tabla 4 presenta los principales resultados que surgen de estimar el modelo (1) a medida que se incorporan controles en forma sucesiva ${ }^{14}$. La columna (1) solo controla por efectos fijos de municipio y de mes. La columna (2) agrega la tendencia pre-tratamiento de los accidentados cada 100 mil habitantes para captar diferencias entre partidos que antes de la llegada del SAME tengan distinta cantidad de accidentes. La columna (3) incorpora controles por regiones sanitarias ${ }^{15}$, para considerar posibles efectos producto de políticas sanitarias

\footnotetext{
${ }^{14}$ Idealmente, sería deseable controlar por tamaño y/o densidad poblacional de los municipios. A tal fin, se cuenta con proyecciones a partir de extrapolar información del Censo Nacional de Población, Hogares y Viviendas de 2010, cuyos cambios mes a mes son marginales en un periodo tan corto de tiempo, de manera que mayormente están controlados por los efectos fijos por municipio.

${ }^{15}$ De acuerdo a la ley provincial 7016, el territorio de la provincia de Buenos Aires está dividido en 12 regiones sanitarias, cuyos límites fueron fijados de acuerdo a factores geográficos, demográficos, socioeconómicos, medios de comunicación y transporte, equipamiento y características sanitarias, entre otros.
} 
comunes a cada región, y de manera similar, la columna (4) controla por zonas viales ${ }^{16}$. Por último, con el fin de controlar por el hecho de que los municipios oficialistas parecen ser más proclives a implementar el SAME (tal como se vio en la sección 3) la columna (5) incluye la dummy Oficialista, que vale 1 si el intendente del partido es oficialista en 2016.

Tabla 4. Estimación del impacto del SAME en las defunciones cada mil accidentados. ${ }^{17}$

\begin{tabular}{lrrrrr}
\hline & \multicolumn{1}{c}{$(\mathbf{1})$} & \multicolumn{1}{c}{$\mathbf{( 2 )}$} & \multicolumn{1}{c}{$(\mathbf{3})$} & \multicolumn{1}{c}{$(\mathbf{4})$} & \multicolumn{1}{c}{$\mathbf{( 5 )}$} \\
\hline SAME & $\mathbf{- 1 5 . 0 1 *}$ & $\mathbf{- 1 5 . 2 8 *}$ & $\mathbf{- 1 6 . 1 4 *}$ & $\mathbf{- 1 6 . 0 4 *}$ & $\mathbf{- 1 6 . 8 9 *}$ \\
& $(8.78)$ & $(8.75)$ & $(8.23)$ & $(8.53)$ & $(9.71)$ \\
Tasa de accidentados pre-trend x EF trimestre & NO & SI & SI & SI & SI \\
Región sanitaria x EF trimestre & NO & NO & SI & SI & SI \\
Zona vial x EF trimestre & NO & NO & NO & SI & SI \\
Oficialista x EF trimestre & NO & NO & NO & NO & SI \\
\hline Observaciones & 4,376 & 4,376 & 4,376 & 4,376 & 4,376 \\
Número de partidos & 135 & 135 & 135 & 135 & 135 \\
\hline
\end{tabular}

Nota: La variable dependiente de todas las columnas es la tasa de defunciones cada mil accidentados, cuya media en la línea de base es 77.87. Los errores estándar robustos se presentan entre paréntesis. Todas las regresiones incluyen efectos fijos por municipio y mes, y dummies que controlan por estacionalidad mensual y anual. $* * * \mathrm{p}<0.01, * * \mathrm{p}<0.05, * \mathrm{p}<0.10$

Como se observa, los resultados son robustos a los cambios en la especificación. El efecto estimado es siempre significativo y su magnitud varía en un rango de -15 a -17 defunciones cada mil accidentados, lo que representa una disminución de $19 \%$ a $21 \%$ de la tasa de defunciones con respecto a la línea de base para los municipios que incorporaron el SAME.

Como se mencionó antes, un supuesto de identificación clave es el de tendencias paralelas, es decir, que en ausencia del tratamiento la tasa de defunciones hubiera evolucionado de la misma forma en el grupo de municipios con y sin SAME (Gertler et al., 2011). Aunque no es posible testear este supuesto, se puede ganar confianza en su validez si se confirma que

\footnotetext{
${ }^{16}$ De acuerdo con la Dirección de Vialidad de la Provincia de Buenos Aires, la provincia se divide en 12 zonas viales. Cada una tiene la potestad de supervisar y aprobar el cumplimiento de las políticas viales que afecten a los partidos de su zona. http://www.vialidad.gba.gov.ar/indexprincipal.php?p=partidosxzonadvba.

${ }^{17} \mathrm{La}$ discrepancia entre las observaciones totales y las utilizadas en la regresión se debe a que en algunos meses no se producen ni defunciones ni accidentes, por lo que la variable dependiente resulta ser 0/0. En la sección 6 se presentan a modo de robustez las estimaciones con la muestra completa, reemplazando por 0 todos los casos donde la variable tiene información faltante.
} 
los municipios tratados y no tratados exhibían una evolución similar de las defunciones previa a la implementación del SAME.

Si bien la evidencia presentada en la Figura 2 sugiere que tanto las tendencias como los niveles son similares en el período pre-tratamiento, para reforzar este hallazgo se testea formalmente que las tendencias sean iguales, estimando un modelo de la tasa de defunciones cada mil accidentados en función de una dummy de tratamiento, efectos fijos por mes y la interacción entre las dummies de mes y de tratamiento, utilizando solamente las observaciones del período pre-tratamiento, esto es, todas las observaciones del grupo de control y solo los meses pre-tratamiento del grupo tratado.

Luego se aplica un test $\mathrm{F}$ para evaluar la hipótesis nula de que en los meses en los que ningún municipio tenía SAME, los coeficientes de las interacciones son conjuntamente iguales a cero, de donde surge que no hay evidencia para rechazar la hipótesis nula. ${ }^{18}$ Por lo tanto, se presume la existencia de una tendencia común para los grupos de tratamiento y control antes de la implementación del SAME, lo que nos permite tener mayor confianza de que los cambios de tendencia post-tratamiento se deben efectivamente a la incorporación del SAME.

\section{Robustez}

En esta sección se realizan una serie de análisis de robustez con el fin de otorgarle mayor validez a los resultados obtenidos anteriormente.

\section{(i) Experimento falso}

El primer ejercicio consiste en un experimento falso o placebo donde se simula que la implementación del SAME ocurrió en algún mes previo al de su implementación real. Si las conclusiones son válidas, no debería haber relación significativa entre el placebo y las defunciones por accidentes viales. La Tabla 5 muestra el resultado de simular que el SAME se implementó de 1 a 6 meses antes de su puesta en marcha verdadera, es decir, se estima el modelo (1) pero con la variable de tratamiento placebo en lugar de la real, utilizando solo las observaciones que no recibieron tratamiento. Se puede observar que los coeficientes

\footnotetext{
${ }^{18}$ El estadístico $\mathrm{F}$ correspondiente es $\mathrm{F}(7,134)=0.63$ con Prob $>\mathrm{F}=0.71$.
} 
asociados a las variables placebo no son significativos en ninguno de los casos, con p-valores muy altos.

Tabla 5. Experimento falso sobre las defunciones cada mil accidentados.

\begin{tabular}{|c|c|c|c|c|c|c|}
\hline Placebo t-1 & $\begin{array}{c}-5.32 \\
(0.71)\end{array}$ & & & & & \\
\hline Placebo t-2 & & $\begin{array}{r}-5.48 \\
(0.66)\end{array}$ & & & & \\
\hline Placebo t-3 & & & $\begin{array}{r}-5.11 \\
(0.67)\end{array}$ & & & \\
\hline Placebo t-4 & & & & $\begin{array}{r}-3.04 \\
(0.80)\end{array}$ & & \\
\hline Placebo t-5 & & & & & $\begin{array}{l}2.07 \\
(0.88)\end{array}$ & \\
\hline Placebo t-6 & & & & & & $\begin{array}{l}8.13 \\
(0.56)\end{array}$ \\
\hline Observaciones & 3,422 & 3,422 & 3,422 & 3,422 & 3,422 & 3,422 \\
\hline Partidos & 135 & 135 & 135 & 135 & 135 & 135 \\
\hline
\end{tabular}

Nota: La variable dependiente de todas las columnas es la tasa de defunciones cada mil accidentados. El p-valor robusto se presenta entre paréntesis. Todas las regresiones incluyen efectos fijos por municipio y mes.

\section{(ii) Impacto del SAME sobre la tasa de accidentados}

En este segundo ejercicio se estudia el impacto del SAME en la tasa de accidentados cada 100 mil habitantes, resultado que no debería verse afectado por la implementación del SAME. Encontrar un "impacto" sobre esta variable pondría en duda el mecanismo detrás de nuestros resultados. En la Tabla 6 se reportan los resultados de estimar el modelo (1) pero reemplazando la variable dependiente por la tasa de accidentados cada 100 mil habitantes. Los coeficientes son muy pequeños y en ningún caso significativos, lo que otorga credibilidad a nuestra estrategia de identificación. 
Tabla 6. Impacto del SAME sobre la tasa de accidentados cada 100 mil habitantes.

\begin{tabular}{lrrrrr}
\hline & $\mathbf{( 1 )}$ & $\mathbf{( 2 )}$ & \multicolumn{1}{c}{$(\mathbf{3})$} & \multicolumn{1}{c}{$(\mathbf{4})$} & \multicolumn{1}{c}{$(\mathbf{5})$} \\
\hline SAME & $\mathbf{0 . 6 6}$ & $\mathbf{0 . 4 7}$ & $\mathbf{0 . 5 7}$ & $\mathbf{1 . 6 8}$ & $\mathbf{1 . 5 4}$ \\
& $(1.14)$ & $(1.06)$ & $(1.20)$ & $(1.29)$ & $(1.52)$ \\
Tasa de accidentados pre-trend x EF trimestre & NO & SI & SI & SI & SI \\
Región sanitaria x EF trimestre & NO & NO & SI & SI & SI \\
Zona vial x EF trimestre & NO & NO & NO & SI & SI \\
Oficialista x EF trimestre & NO & NO & NO & NO & SI \\
\hline Observaciones & 4,860 & 4,860 & 4,860 & 4,860 & 4,860 \\
Número de partidos & 135 & 135 & 135 & 135 & 135 \\
\hline
\end{tabular}

Nota: La variable dependiente de todas las columnas es la tasa de accidentados cada 100 mil habitantes. Todas las regresiones incluyen efectos fijos por municipio y mes. Errores estándar robustos entre paréntesis $* * * \mathrm{p}<0.01, * * \mathrm{p}<0.05, * \mathrm{p}<0.10$

\section{(iii) Robustez a la definición de la muestra}

Como ya se mencionó en la sección anterior, la variable dependiente presenta observaciones con información faltante debido a que hay meses en donde no se produce ningún accidente. En esta sección se imputa un valor a esas observaciones para contar con un panel balanceado de municipios durante todo el periodo de análisis. En particular, se asigna un valor igual a 0 cuando la tasa de defunciones resulta ser 0/0, suponiendo que en ese mes el municipio en cuestión tuvo al menos un lesionado en lugar de ninguno. ${ }^{19} \mathrm{La}$ Tabla 7 muestra que los resultados se mantienen luego de este cambio en la definición de la muestra. También se mantiene la robustez de los resultados a todos los chequeos anteriores (tendencias paralelas y ejercicios placebo $)^{20}$, ambas pruebas validan los efectos encontrados.

\footnotetext{
${ }^{19}$ Este supuesto no parece fuerte, ya que indica que en los partidos más pequeños en donde no se registraron lesionados a lo largo de todo un mes, posiblemente se haya producido al menos una colisión de menor gravedad que no haya requerido intervención policial.

${ }^{20}$ El estadístico $\mathrm{F}$ correspondiente al testeo de tendencias paralelas es $\mathrm{F}(7,134)=0.78$ con Prob $>\mathrm{F}=0.59$. Los demás resultados están disponibles a solicitud.
} 
Tabla 7. El impacto del SAME en las defunciones cada mil accidentados, muestra completa.

\begin{tabular}{lrrrrr}
\hline & \multicolumn{1}{c}{$(\mathbf{1})$} & \multicolumn{1}{c}{$(\mathbf{2})$} & \multicolumn{1}{c}{$(\mathbf{3})$} & \multicolumn{1}{c}{$\mathbf{4})$} & \multicolumn{1}{c}{$\mathbf{( 5 )}$} \\
\hline SAME & $\mathbf{- 1 4 . 2 4 *}$ & $\mathbf{- 1 4 . 6 5 *}$ & $\mathbf{- 1 5 . 9 3 * *}$ & $\mathbf{- 1 6 . 6 7 * *}$ & $\mathbf{- 1 8 . 4 4 * *}$ \\
& $(7.70)$ & $(7.76)$ & $(7.58)$ & $(7.68)$ & $(8.36)$ \\
Tasa de accidentados pre-trend x EF trimestre & NO & SI & SI & SI & SI \\
Región sanitaria x EF trimestre & NO & NO & SI & SI & SI \\
Zona vial x EF trimestre & NO & NO & NO & SI & SI \\
Oficialista x EF trimestre & NO & NO & NO & NO & SI \\
\hline Observaciones & 4,860 & 4,860 & 4,860 & 4,860 & 4,860 \\
Número de partidos & 135 & 135 & 135 & 135 & 135 \\
\hline
\end{tabular}

Nota: La variable dependiente de todas las columnas es la tasa de defunciones cada mil accidentados, cuya media en la línea de base es 76.4. Los errores estándar robustos se presentan entre paréntesis. Todas las regresiones incluyen efectos fijos por municipio y mes, y dummies que controlan por estacionalidad mensual y anual. $* * * \mathrm{p}<0.01, * * \mathrm{p}<0.05, * \mathrm{p}<0.10$

\section{(iv) Descomposición de Goodman-Bacon.}

En esta sección se implementa la descomposición propuesta por Goodman-Bacon (2018) para responder una pregunta común en la práctica: cuánto del efecto promedio proviene de la comparación entre tratados y no tratados, y cuánto de la secuencialidad del tratamiento. Goodman-Bacon (2018) demuestra que el estimador de diferencias en diferencias de una regresión lineal de efectos fijos es un promedio ponderado de todos los estimadores posibles que surgen de comparar grupos de tratamiento y control que van cambiando en el tiempo cuando el tratamiento es secuencial. En particular, realiza tres comparaciones: (1) unidades tratadas versus unidades no tratadas, (2) unidades tratadas "temprano" como grupo de tratamiento versus unidades tratadas "tarde" como grupo de control (antes de que comience su tratamiento), y (3) unidades tratadas "temprano" como control versus "tarde" como tratamiento, una vez que comienza el tratamiento de estas últimas. Como en cualquier estimador de mínimos cuadrados, las ponderaciones de cada comparación son proporcionales a los tamaños de los grupos y a la varianza del tratamiento. ${ }^{21,22}$

\footnotetext{
${ }^{21}$ La variación del tratamiento es más alta para los grupos tratados en la mitad del panel y más baja para los grupos tratados en los extremos.

${ }^{22}$ Para más detalle, en el anexo E se explica formalmente la metodología de la descomposición.
} 
La Tabla 8 es el resultado de aplicar la descomposición de Goodman-Bacon al impacto del SAME estimado con el modelo lineal de efectos fijos. ${ }^{23}$

Tabla 8. Descomposición del efecto promedio del SAME sobre la tasa de defunciones.

\begin{tabular}{lll}
\hline DD lineal con efectos fijos: & $\mathbf{- 1 4 . 2 4 *}$ & \\
\hline Descomposición & Beta & Peso \\
\hline $\mathrm{T}=$ "temprano" vs. C = "tarde" & $-23.46 * * *$ & 0.33 \\
$\mathrm{~T}=$ "tarde" vs. C = "temprano" & $-26.98 * * *$ & 0.11 \\
Tratados vs. Nunca tratados & -6.19 & 0.56 \\
\hline
\end{tabular}

Nota: $\mathrm{T}=$ tratamiento, $\mathrm{C}=$ control. $\mathrm{La}$ variable dependiente es la tasa de defunciones cada mil accidentados con muestra completa presentada en la sub-sección anterior. Se utiliza esta variable debido a que la descomposición funciona cuando el panel está perfectamente balanceado. Así, se replica efecto encontrado en la columna (1) de la Tabla 7. En cuanto a la inferencia estadística, el trabajo de Goodman-Bacon no aborda este tema, por lo tanto se estimó el error estándar de los coeficientes por el método de bootstrap con 200 repeticiones y cluster por municipio.

Se puede ver que el efecto encontrado en el resultado principal $\left(\hat{\beta}^{D D}=-14.24\right)$ es un promedio ponderado de los tres subgrupos. Además, sumar los pesos de las comparaciones de tiempo ("tarde" vs. "temprano" y "temprano" vs. "tarde”) indica exactamente cuánto de $\hat{\beta}^{D D}$ proviene de la secuencialidad temporal del tratamiento, en este caso un $44 \%$.

Intuitivamente, este resultado se puede explicar con la evidencia presentada en la Figura 2: allí se observa que la tendencia de la tasa de defunciones de los municipios tratados y no tratados es similar hasta mediados de 2017, momento en el cual comienza a verse una brecha creciente hasta el final del periodo. Esto es consistente con el carácter secuencial de la implementación e indica que el efecto del SAME en la provincia comienza a notarse aproximadamente un año después de su llegada, posiblemente por un efecto de escala y/o de composición de grupos. Notar además que el valor promedio de la brecha (cercano a -23) es similar a los efectos de las comparaciones de tiempo encontrados en la descomposición (-23 y -26). De esta manera, el efecto promedio es un resumen que esconde cierta heterogeneidad proveniente de la secuencialidad del tratamiento.

\footnotetext{
${ }^{23}$ Afortunadamente se encuentra disponible un comando (bacondecomp) para aplicar esta descomposición en STATA.
} 
Por último, cuando los efectos del tratamiento varían con el tiempo, las unidades ya tratadas podrían no ser un buen grupo de control, y entonces el resultado promedio estaría sesgado. De ser este el caso, la descomposición anterior permite computar una cota inferior del efecto, que resulta ser de -11.15 , y surge de sustraerle al efecto total la comparación $\mathrm{T}=$ "tarde", $\mathrm{C}=$ "temprano" (ver Goodman-Bacon, 2018).

\section{Conclusiones}

A finales de 2016 la provincia de Buenos Aires comenzó a implementar el SAME, un servicio de atención médica de emergencias gratuito y con un número telefónico único para todo el territorio, con el objetivo brindar a toda la población acceso a una atención eficiente y de calidad ante situaciones de emergencias. Para diciembre de 2017 la provincia contaba con un $27 \%$ de los municipios adheridos a este servicio, y para fines de 2018 este porcentaje ascendía a $64 \%$, lo que a su vez representa un $48 \%$ de la población bonaerense para 2017 y un $68 \%$ para 2018.

La hipótesis de este trabajo es que a partir de la incorporación del SAME, las muertes por accidentes viales deberían bajar, debido a lo que se conoce en medicina como "la hora dorada", en donde los plazos de atención médica luego de un traumatismo pueden marcar la diferencia entre la vida y la muerte. Una llegada rápida y eficaz de las ambulancias al lugar del hecho, junto con una adecuada atención pre-hospitalaria dentro de la ambulancia puede reducir significativamente la probabilidad de muerte y de discapacidad por lesiones graves en los accidentados. Utilizando la metodología de Diferencias en Diferencias se encontró que la implementación del SAME reduce en promedio un 19\% las muertes cada mil accidentados respecto de la línea de base. Este resultado es significativo y robusto ante distintas especificaciones.

Si bien la metodología no permite identificar los mecanismos que hay detrás de este efecto, es posible mencionar de manera no exhaustiva algunos de los cuales podrían estar operando alternativa o simultáneamente: en primer lugar, el carácter integral de este servicio de emergencias, con protocolos estandarizados, métodos de atención ampliamente reconocidos en medicina de emergencias y con alcance universal y gratuito, es algo que no existía hasta 
el momento en la provincia. De modo que antes de la llegada del SAME, las víctimas de un accidente de tránsito debían esperar la llegada de ambulancias privadas (si es que accedían a este servicio), trasladarse de manera particular hacia el hospital, o en algunos casos aguardar por un servicio público que no tenía las características del SAME. ${ }^{24}$

Asimismo, el tiempo de demora de las ambulancias (de 10 a 12 minutos según el protocolo de atención), también da cuenta de que podría estar operando el mecanismo de "la hora dorada". Por otra parte, la inversión en publicidad mediante la colocación de llamativos carteles en rutas, autopistas y espacios públicos donde se notifica a la población de la existencia de este servicio gratuito, universal y con un número fácil de recordar, podría ayudar a que al momento de producirse un accidente vial, las víctimas y/o los testigos puedan pedir auxilio rápidamente llamando a las ambulancias con el número que figura en los carteles.

Por último, un mecanismo más marginal podría ser que el SAME contribuya a mejorar la coordinación entre municipios, por ejemplo en casos donde los accidentes ocurren en el límite entre dos municipios y no está claro cuál debe acudir, el SAME con sus bases organizadas y comunicadas entre sí, podría determinar rápidamente cuál de ellas debe encargarse de asistir a las víctimas según la ubicación de donde proviene la llamada.

Estos son solo algunos de los mecanismos que podrían estar operando detrás del resultado encontrado, pero queda para futuras investigaciones evaluar su relevancia mediante evidencia empírica. Sin embargo, la reducción encontrada en las muertes por accidentes viales es contundente y por lo tanto concluimos que sería deseable expandir el SAME a otros municipios y provincias.

\footnotetext{
${ }^{24}$ Solo en unos pocos municipios se contaba con un servicio público de emergencias (SIES), del cual solo se sabe que no era un servicio de las características del SAME.
} 


\section{Referencias}

Agencia Nacional de Seguridad Vial (2019). Anuario Estadístico de siniestralidad vial. Informe de la Dirección Nacional de Observatorio Vial, Ministerio de Transporte.

Angrist, J. D., \& Pischke, J. S. (2008). Mostly harmless econometrics: An empiricist's companion. Princeton university press.

Bentham, G., (1986). Proximity to hospital and mortality from motor vehicle traffic accidents. Social Science and Medicine 23 (10), 1021-1026.

Brodsky, H., (1990). Emergency medical service rescue time in fatal road accidents. Transportation Research Records 1270, 89-96.

Brodsky, H., (1992). Delay in ambulance dispatch to road accidents. American Journal of Public Health 82, 873-875.

Brodsky, H., (1993). The call for help after an injury road accident. Accident Analysis and Prevention $25,123-130$.

Cameron, A. C., \& Trivedi, P. K. (2005). Microeconometrics: methods and applications. Cambridge university press.

Card, D. (1990). “The Impact of the Mariel Boatlift on the Miami Labor Market”. Industrial and Labor Relations Review 43(2): 245-257.

Card, D. y Krueger, A. (1994). "Minimum Wages and Employment: A case of Study of the Fast-Food industry in New Jersey and Pennsylvania”. American Economic Review 84: 772- 793.

Castillo-Manzano, J. I., Castro-Nuño, M., \& Fageda, X. (2014). Can health public expenditure reduce the tragic consequences of road traffic accidents? The EU-27 experience. The European Journal of Health Economics, 15(6), 645-652.

Clark, D.E., Cushing, B.M., (2002). Predicted effect of automatic crash notification on traffic mortality. Accident Analysis and Prevention 34, 507-513.

Clark DE, Qian J, Sihler KC, Hallagan LD, Betensky RA (2012). The distribution of survival times after injury. World J. Surg., 36 (7), pp. 1562-1570.

Clark, D.E., Winchella, R.J., and Betensky, R.A (2013). Estimating the effect of emergency care on early survival after traffic crashes, Accident Analysis \& Prevention, Volume 60, Issue 0, pp 141-147. 
Dirección Provincial de Estadística (2019). Estadísticas de incidentes viales con fallecidos y lesionados de la provincia de Buenos Aires. Informe de la Dirección Provincial de Estadística del Ministerio de Economía de Buenos Aires.

Durkin, M.,McElroy, J., Guan, H., Bigelow, W., Brazelton, T., 2005. Geographic analysis of traffic injury in Wisconsin: impact on case fatality of distance to Level I/II trauma care. Wisconsin Medical Journal 104, 26-31.

Evanco, W.M., 1999. The potential impact of rural mayday systems on vehicular crash fatalities. Accident Analysis and Prevention 31, 455-462.

Gertler, P. J., Martinez, S., Premand, P., Rawlings, L. B., \& Vermeersch, C. M. (2011). Impact evaluation in practice. The World Bank.

Goodman-Bacon, A. (2018). Difference-in-differences with variation in treatment timing (No. w25018). National Bureau of Economic Research.

Iserson, K. V., \& Moskop, J. C. (2007). Triage in medicine, part I: concept, history, and types. Annals of emergency medicine, 49(3), 275-281.

Jacobs, G., Aeron-Thomas, A., \& Astrop, A. (2000). Estimating Global Road Fatalities. Transport Research Laboratory. London.

Li, M.D., Doong, J.L., Chang, K.K., Lu, T.H., Jeng, M.C., 2008. Differences in urban and rural accident characteristics and medical service utilization for traffic fatalities in less-motorized societies. Journal of Safety Research 39, 623-630.

Ministerio de Salud de la Provincia de Buenos Aires, 2017. Manual de Atención de Emergencias Médicas de SAME Provincia de Buenos Aires. Documento interno.

Muelleman, R.L., Mueller, K., 1996. Fatal motor vehicle crashes: variations of crash characteristics within rural regions of different population densities. The Journal of Trauma: Injury, Infection and Critical Care 41, 315-320.

Murad, M. K., Issa, D. B., Mustafa, F. M., Hassan, H. O., \& Husum, H. (2012). Prehospital trauma system reduces mortality in severe trauma: a controlled study of road traffic casualties in Iraq. Prehospital and disaster medicine, 27(1), 36-41.

Noland, R.B., (2003). Medical treatment and traffic fatality reductions in industrialized countries. Accident Analysis and Prevention 35, 877-883. 
Noland, R.B., Quddus, M.A., (2004). Improvements in medical care and technology and reductions in traffic-related fatalities in Great Britain. Accident Analysis and Prevention 36, 103-113.

Sanchez-Mangas, R., Garcia-Ferrrer, A., de Juan, A., Arroyo, A.M., 2010. The probability of death in road traffic accidents. How important is a quick medical response? Accid. Anal. Prev. 42 (4), 10481056.

Travis, L.L., Clark, D.E., Haskins, A.E., Kilch, J.A., 2012. Mortality in rural locations after severe injuries from motor vehicle crashes. J. Safety Res. 43 (5-6), 375-380.

Vasudevan, V., Singh, P., \& Basu, S. (2016). Importance of awareness in improving performance of emergency medical services (EMS) systems in enhancing traffic safety: A lesson from India. Traffic injury prevention, 17(7), 699-704.

World Health Organization. (2015). Global status report on road safety 2015. World Health Organization.

World Health Organization (2017). 10 datos sobre la seguridad vial en el mundo. Disponible en: https://www.who.int/features/factfiles/roadsafety/es/

World Health Organization (2018). Global status report on road safety 2018. Geneva: Licence: CC BYNC- SA 3.0 IGO.

Zwerling, C., Peek-Asa, C., Whitten, P.S., Choi, S.W., Sprince, N.L., Jones, M.P., 2005. Fatal motor vehicles crashes in rural and urban areas: decomposing rates into contributing factors. Injury Prevention 11, 24-28. 


\section{Anexo A. Estadísticas descriptivas sobre accidentes viales en la provincia de Buenos Aires.}

En el siguiente apartado se presentan algunas estadísticas provenientes de la base de microdatos de defunciones para los años 2016, 2017 y 2018, en donde se puede caracterizar los fallecimientos según edad, sexo, mes, tipo de víctima, tipo de vía, y vehículo. ${ }^{25}$

En primer lugar, se puede ver en la Tabla A.1 que los datos muestran un leve patrón estacional, con aumentos a finales del invierno y picos en los meses de verano y de semana santa (en abril) lo que podría asociarse a la mayor movilidad durante esos meses y, por tanto, a un aumento en la magnitud de la población expuesta al riesgo. Sin embargo, a menos que se estimen tasas de víctimas fatales, no es posible afirmar un mayor riesgo (por unidad de exposición) en esos meses.

Por otra parte, en la Tabla A.2 y la Figura A.1 se puede observar que la mayor parte de las víctimas son varones de 15 a 29 años, representando el $28 \%$ de los fallecidos totales. Además, la cantidad total de varones fallecidos más que triplica a la de las mujeres.

Asimismo, en la Tabla A.3 se puede ver que la gran mayoría de las muertes ocurre en calles y rutas (48\% y $35 \%$ respectivamente), aunque no es posible concluir la magnitud del riesgo diferencial a menos que se controle por la exposición al riesgo en cada caso.

Además, un $32 \%$ de las defunciones corresponden a motociclistas, seguido de autos (17\%) y peatones $(7 \%)$, como se puede ver en la Tabla A.4. Dado que la cantidad de personas que viajan en moto es inferior a la que viaja en automóvil, es posible afirmar que la mayor cantidad de víctimas fatales en incidentes con motos se debe al mayor riesgo asociado a este tipo de vehículo (DPE, 2019).

Por último, cuando se analiza la distribución por tipo de víctima en la Tabla A.5, se observa que la mayor cantidad de muertes se da entre los conductores (48\%), seguido de un $15 \%$ de acompañantes.

\footnotetext{
${ }^{25}$ Este tipo de caracterización solo es posible para la base de datos de defunciones, y no en la de lesionados.
} 
Tabla A.1. Número de defunciones por accidentes viales por año y mes.

\begin{tabular}{lrrr}
\hline Mes & $\mathbf{2 0 1 6}$ & $\mathbf{2 0 1 7}$ & $\mathbf{2 0 1 8}$ \\
\hline Enero & 151 & 117 & 107 \\
Febrero & 108 & 90 & 131 \\
Marzo & 111 & 102 & 106 \\
Abril & 102 & 123 & 119 \\
Mayo & 119 & 102 & 136 \\
Junio & 111 & 102 & 119 \\
Julio & 98 & 102 & 106 \\
Agosto & 111 & 129 & 88 \\
Septiembre & 99 & 125 & 112 \\
Octubre & 88 & 122 & 96 \\
Noviembre & 119 & 114 & 101 \\
Diciembre & 109 & 140 & 123 \\
\hline Total & $\mathbf{1 3 2 6}$ & $\mathbf{1 3 6 8}$ & $\mathbf{1 3 4 4}$ \\
\hline
\end{tabular}

Fuente: elaboración propia en base a Observatorio Vial de la Provincia de Buenos Aires.

Tabla A.2. Cantidad de defunciones por edad y sexo, 2016-2018.

\begin{tabular}{lrr}
\hline Edad & Mujeres & Hombres \\
\hline $0-14$ & 55 & 106 \\
$15-29$ & 237 & 1127 \\
$30-44$ & 137 & 704 \\
$45-54$ & 76 & 311 \\
$55-64$ & 102 & 271 \\
$65+$ & 201 & 352 \\
Sin determinar & 70 & 279 \\
\hline Total & $\mathbf{8 7 8}$ & $\mathbf{3 1 5 0}$ \\
\hline
\end{tabular}

Fuente: elaboración propia en base a Observatorio Vial de la Provincia de Buenos Aires. Nota: Hay 10 fallecimientos sin determinar sexo ni edad, por lo tanto el total no coincide con el resto de las tablas.

Figura A.1. Histograma de defunciones por edad y sexo.

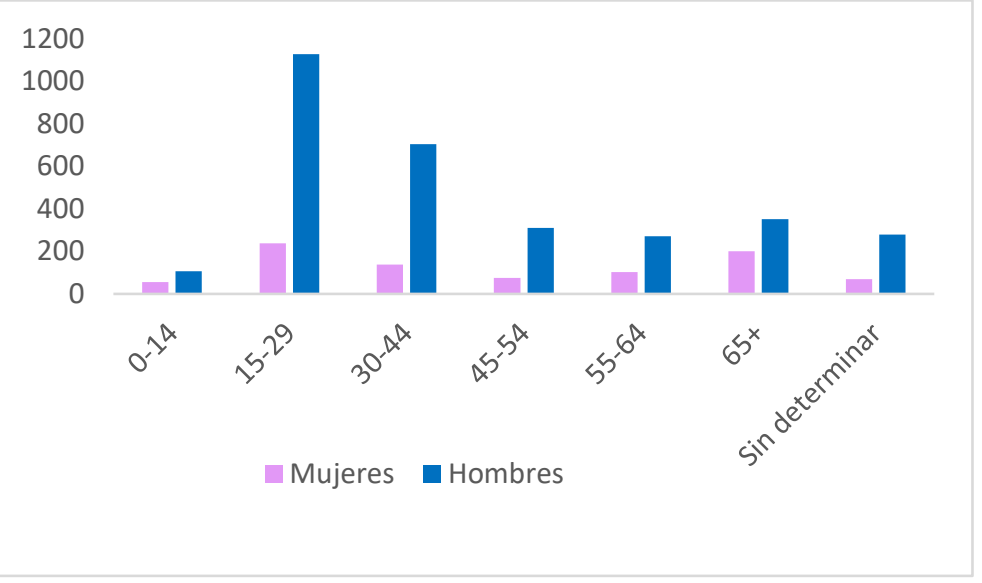

Fuente: elaboración propia en base a Observatorio Vial de la Provincia de Buenos Aires 
Tabla A.3. Fallecidos por tipo de vía. 2016-2018

\begin{tabular}{lrr}
\hline Tipo de vía & Cantidad & \% \\
\hline Autopista & 221 & $5 \%$ \\
Calle & 1921 & $48 \%$ \\
Ruta & 1428 & $35 \%$ \\
Sin determinar & 468 & $12 \%$ \\
\hline Total & $\mathbf{4 0 3 8}$ & $\mathbf{1 0 0 \%}$ \\
\hline
\end{tabular}

Fuente: elaboración propia en base a Observatorio Vial de la Provincia de Buenos Aires.

Tabla A.4. Fallecidos por tipo de vehículo. 2016-2018

\begin{tabular}{lrr}
\hline Tipo de vehículo & Cantidad & \% \\
\hline Moto & 1305 & $32 \%$ \\
Auto & 705 & $17 \%$ \\
Camioneta & 229 & $6 \%$ \\
Camión & 74 & $2 \%$ \\
Colectivo & 22 & $1 \%$ \\
Bicicleta & 149 & $4 \%$ \\
Peatón & 292 & $7 \%$ \\
Otro & 2 & $0 \%$ \\
Sin determinar & 1260 & $31 \%$ \\
\hline Total & $\mathbf{4 0 3 8}$ & $\mathbf{1 0 0 \%}$ \\
\hline
\end{tabular}

Fuente: elaboración propia en base a Observatorio Vial de la Provincia de Buenos Aires.

Tabla A.5. Cantidad de fallecidos por tipo de víctima. 2016-2018

\begin{tabular}{lrr}
\hline Tipo de víctima & Cantidad & \% \\
\hline Conductor & 1951 & $48 \%$ \\
Acompañante & 607 & $15 \%$ \\
Peatón & 466 & $12 \%$ \\
Pasajero & 22 & $1 \%$ \\
Sin determinar & 992 & $25 \%$ \\
\hline Total & $\mathbf{4 0 3 8}$ & $\mathbf{1 0 0 \%}$ \\
\hline
\end{tabular}

Fuente: elaboración propia en base a Observatorio Vial de la Provincia de Buenos Aires. 


\section{Anexo B. Protocolos de atención y equipamiento de las ambulancias del SAME.}

\section{Protocolos de atención.}

Tabla B.1. Clasificación de prioridades en el sistema de triage.

\begin{tabular}{cl}
\hline Código & Caso \\
\hline Rojo & $\begin{array}{l}\text { Patología sanitaria con repercusión vital, incidente grave con heridos, } \\
\text { situaciones con grave riesgo para el personal actuante, atentados, } \\
\text { incendios, solicitud de cualquier miembro de dotación actuante o } \\
\text { apoyo a cualquier recurso, incidentes en los que se prevean grandes } \\
\text { lesiones, intento de suicidio no traumático, enfermos psiquiátricos } \\
\text { violentos con riesgo para las personas }\end{array}$ \\
\hline Amarillo & $\begin{array}{l}\text { Patología sanitaria de urgencia no vital, apertura de puerta por } \\
\text { bomberos, incidente sin especificar o sin confirmar heridos graves }\end{array}$ \\
\hline Verde & $\begin{array}{l}\text { Patología sanitaria de consulta, información de problema social, } \\
\text { solicitudes de psiquiatría o enfermos psiquiátricos no violentos. }\end{array}$ \\
\hline Negro & $\begin{array}{l}\text { Se debe realizar la constatación del óbito. No se debe mover el cuerpo } \\
\text { del paciente en caso de muerte dudosa. No se confecciona certificado } \\
\text { de defunción. Se debe dar intervención policial. }\end{array}$ \\
\hline Fuente: Manual de Atención de Emergencias Médicas de SAME Provincia de Buenos Aires.
\end{tabular}

\section{Equipamiento de las ambulancias.}

Cada ambulancia del SAME cuenta con un amplio equipamiento y diversos insumos médicotecnológicos, los cuales se listan a continuación.

Bolso de vía aérea: contiene un set de cánulas orofaríngeas, laringoscopio con cuatro ramas y alimentación con dos baterías de alcalina, máscara laríngea descartable, guía de eschmann descartable, oxímetro de pulso, set de tubos endrotraqueales, tubo combinado esófagotraqueal, fijador de tubos endotraqueal, aspirador manual, fastrach descartable, set de cricostomia, bolsa válvula con mascarilla con reservorio adulto, bolsa válvula con mascarilla con reservorio pediátrico, mascarilla tipo venturi, mascarilla para nebulizar, guantes descartables, jeringas de $10 \mathrm{~cm} 3$, pinza de maguil adulto, antiparras, tres resucitadores descartables (adulto, pediátrico, neonatal).

Bolso de trauma: cuenta con una venda tipo cambric orillada, un apósito gasa de 10x20, una venda elástica, una venda elástica adherente, una esponja hemostática celulosa, equipo de 
admisión micro y macro goteo, solución fisiológica por $500 \mathrm{ml}$, solución dextrosa al 5\% por $500 \mathrm{ml}$, solución ringer lactato por $500 \mathrm{ml}$, jeringa descartable de $10 \mathrm{ml}$, jeringa descartable de $20 \mathrm{ml}$, una caja de guantes de examinación medianos, un collar tipo filadelfa chico, uno mediano, uno grande y uno extra grande, un set de férulas inflables, una tijera de trauma multipropósito, una manta térmica, un inflador para férulas, catéter para acceso intravenoso número 16, 18, 20, 22 у 24.

Bolso de parto: incluye una manta térmica, un clamp para cordón umbilical, diez apósitos estériles, una compresa estéril, set de taponajes vaginales, un apósito post parto, una caja de veinte tampones, un guante estéril (un par), un par de antiparras, un camisolín.

Bolso de Quemado: contiene sábanas estériles y dos descartables, una manta térmica, diez apósitos estériles, diez jeringas de $10 \mathrm{~cm} 3$, diez jeringas de $20 \mathrm{~cm} 3$, una compresa estéril, diez agujas hipo alergénicas, una solución fisiológica, un guante estéril.

Además, las ambulancias cuentan con un maletín de medicación, aspirador a diafragma con batería y cargador, sistema de monitoreo de glucosa en sangre, inmovilizador adulto latero cervical, inmovilizador pediátrico latero cervical, tensiómetro aneroide con estetoscopio, un chaleco de inmovilización adulto, un chaleco de inmovilización pediátrico, dos tubos de oxígeno ultraliviano con manómetro, un tubo de oxígeno ultraliviano mochila, una camilla con cinturones de seguridad, una silla de ruedas, un panel central de oxígeno, un cardiodesfribilador con monitor. 


\section{Anexo C. Definición de las variables}

\begin{tabular}{|c|c|c|}
\hline Variable & Definición & Fuente \\
\hline Accidentados & $\begin{array}{l}\text { Suma de lesionados y fallecidos en accidentes } \\
\text { viales }\end{array}$ & $\begin{array}{l}\text { Ministerio de Salud de la PBA, Dirección } \\
\text { Provincial de Estadística de la PBA, } \\
\text { Observatorio Vial de la PBA. }\end{array}$ \\
\hline Tasa de defunciones & $\begin{array}{l}\text { Defunciones cada mil accidentados en siniestros } \\
\text { viales }\end{array}$ & $\begin{array}{l}\text { Ministerio de Salud de la PBA, Dirección } \\
\text { Provincial de Estadística de la PBA, } \\
\text { Observatorio Vial de la PBA. }\end{array}$ \\
\hline Tasa de accidentados & Accidentados cada 100 mil habitantes & $\begin{array}{l}\text { Ministerio de Salud de la PBA, Dirección } \\
\text { Provincial de Estadística de la PBA, } \\
\text { Observatorio Vial de la PBA y Censo Nacional } \\
\text { de Población, Hogares y Viviendas 2010, } \\
\text { INDEC. }\end{array}$ \\
\hline Oficialista & $\begin{array}{l}\text { Variable dummy que vale } 1 \text { si el gobierno } \\
\text { municipal es del mismo partido político que el } \\
\text { gobierno provincial en } 2016 \text { y } 0 \text { en caso contrario }\end{array}$ & Junta Electoral de la Provincia de Buenos Aires \\
\hline Conurbano & $\begin{array}{l}\text { Variable dummy que identifica los } 24 \text { partidos } \\
\text { que rodean a la ciudad de Buenos Aires }\end{array}$ & INDEC \\
\hline NBI & $\begin{array}{l}\text { Porcentaje de hogares con al menos una NBI en } \\
2010 \text { por municipio }\end{array}$ & $\begin{array}{l}\text { Censo Nacional de Población Hogares y } \\
\text { Vivienda } \\
\text { 2010; INDEC. }\end{array}$ \\
\hline Obra social & $\begin{array}{l}\text { Porcentaje de población cubierta con obra social } \\
\text { o prepaga en } 2010 \text { por municipio. }\end{array}$ & $\begin{array}{l}\text { Censo Nacional de Población Hogares y } \\
\text { Vivienda } \\
\text { 2010; INDEC. }\end{array}$ \\
\hline Obras viales en rutas & $\begin{array}{l}\text { Variable dummy que vale } 1 \text { si el municipio fue } \\
\text { afectado por obras viales provinciales en rutas } \\
\text { que se llevaron a cabo durante } 2017-2018 \text {, y } 0 \text { en } \\
\text { caso contrario. }\end{array}$ & $\begin{array}{l}\text { Ministerio de Infraestructura de la Provincia de } \\
\text { Buenos Aires. }\end{array}$ \\
\hline Población (log) & $\begin{array}{l}\text { Logaritmo de la cantidad de habitantes del } \\
\text { municipio. }\end{array}$ & $\begin{array}{l}\text { Censo Nacional de Población y Vivienda } \\
\text { 2010; INDEC. }\end{array}$ \\
\hline $\begin{array}{l}\text { Densidad } \\
\text { poblacional }\end{array}$ & $\begin{array}{l}\text { Cantidad de habitantes por kilómetro cuadrado } \\
\text { del municipio. }\end{array}$ & $\begin{array}{l}\text { Censo Nacional de Población y Vivienda } \\
\text { 2010; INDEC. }\end{array}$ \\
\hline Superficie & $\begin{array}{l}\text { Superficie en kilómetros cuadrados de cada } \\
\text { municipio }\end{array}$ & INDEC \\
\hline Región sanitaria & $\begin{array}{l}\text { Variable categórica que indica a cuál de las } 12 \\
\text { regiones sanitarias de la provincia pertenece el } \\
\text { municipio. }\end{array}$ & $\begin{array}{l}\text { Ministerio de Salud de la Provincia de Buenos } \\
\text { Aires }\end{array}$ \\
\hline Zona vial & $\begin{array}{l}\text { Variable categórica que indica a cuál de las } 12 \\
\text { zonas viales de la provincia pertenece el } \\
\text { municipio. }\end{array}$ & $\begin{array}{l}\text { Dirección de Vialidad de la Provincia de } \\
\text { Buenos Aires }\end{array}$ \\
\hline
\end{tabular}




\section{Anexo D. Robustez}

En esta sección se presentan dos alternativas de la Figura 3, eligiendo un soporte común de partidos y ventanas de tiempo distintas. Se observa que, al igual que en la Figura 3, a partir de la implementación del SAME (barra roja), las defunciones disminuyen y los lesionados aumentan.

\section{Figura D.1. Evolución de las defunciones y lesionados en partidos con SAME alrededor del mes de su implementación.}

Panel A. 56 partidos. Ventana de 3 meses

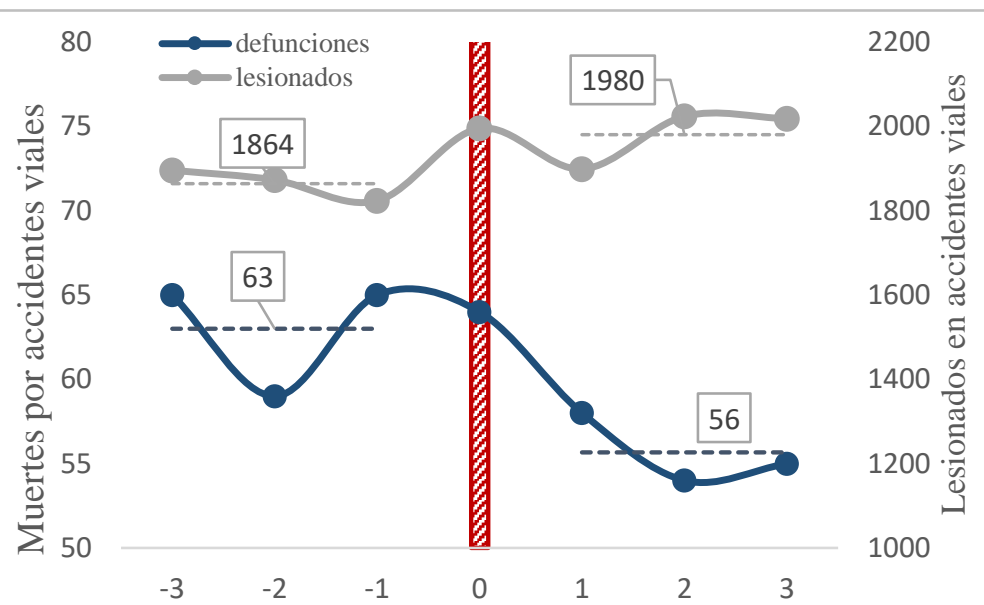

Meses anteriores / posteriores a la implementación del SAME
Panel B. 54 partidos. Ventana de 4 meses.

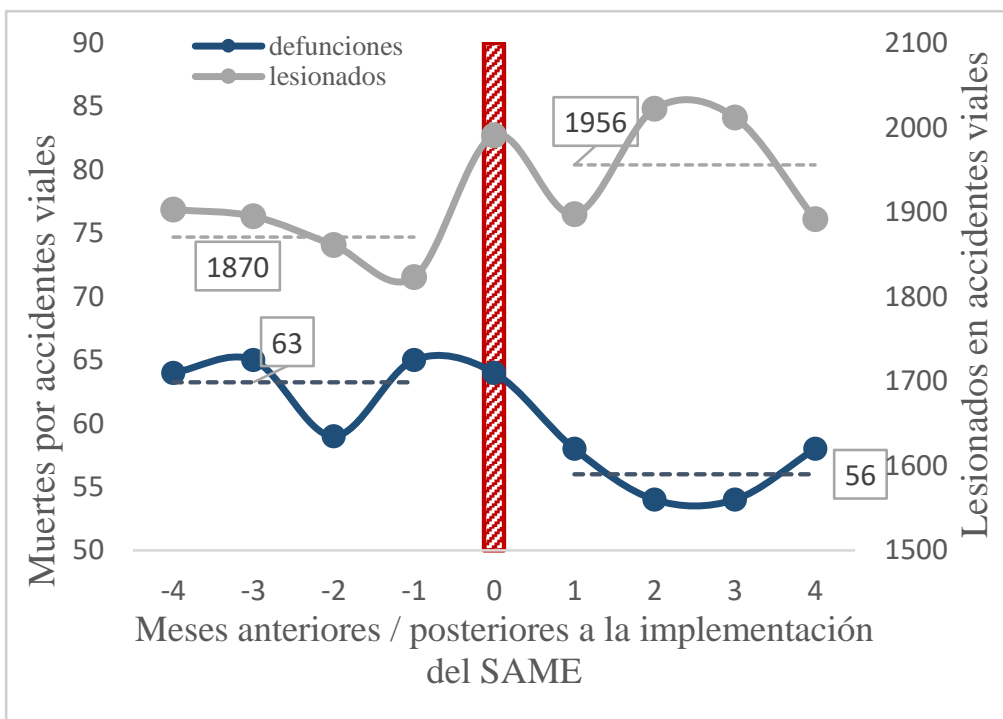

Nota: las líneas punteadas indican el promedio de cada variable antes y después de la implementación del SAME. Naturalmente la variable temporal está definida sólo para aquellos municipios que hayan implementado el servicio. A su vez se elige una ventana de tiempo $t \in(-\mathrm{T}, \mathrm{T})$ para contar con un soporte común de municipios, debido a que el rango que toma $t$ para cada municipio es distinto. Más específicamente, de los 87 municipios que implementaron SAME en el período relevante, en el panel A se realiza una submuestra de 56 municipios $t \in(-3,3)$, y en el panel B se realiza una submuestra de 54 municipios en $t \in(-4,4)$. 


\section{Anexo E. Descomposición de Goodman Bacon.}

La discusión que motiva el trabajo de Goodman-Bacon se centra en comparar el método de diferencias en diferencias clásico con dos períodos y dos grupos, en donde el tratamiento ocurre en el segundo período para uno de esos grupos (DD 2x2), y el método de diferencias en diferencias con tratamiento secuencial. Es bien sabido que el estimador de DD 2x2 es numéricamente equivalente al estimador de una regresión lineal de efectos fijos, pero desafortunadamente, esta equivalencia no se generaliza al diseño de Diferencias en Diferencias (DD) con múltiples períodos de tratamiento. Sin embargo, el estimador lineal con efectos fijos es de uso extendido también para los diseños de DD con tratamiento secuencial (Angrist y Pischke, 2009; Cameron y Trivedi 2005).

Goodman-Bacon muestra que cuando el efecto del tratamiento varía con el tiempo, resumir los resultados con un estimador DD 2x2 podría ser engañoso. En esta línea, propone una forma sencilla de ilustrar cómo funciona la secuencialidad del tratamiento, considerando un panel equilibrado con $T$ períodos $(t)$ y $N$ unidades $(i)$ divididas en tres grupos: los no tratados, $U$; los tratados "temprano", $k$, que reciben un tratamiento binario en $t_{k}^{*}$; y los tratados "tarde", $\ell$, que reciben el tratamiento binario en $t_{l}^{*}>t_{k}^{* 26}$. Cada grupo tiene un tamaño $n_{j}$, con $j=$ $k, l, U ;$ y se denota con $\bar{D}_{j}$ a la proporción de tiempo que cada grupo pasó tratado.

A partir de este esquema, el resultado principal del trabajo de Goodman-Bacon es que el estimador DD de efectos fijos en un diseño con tratamiento secuencial, es un promedio ponderado de todos los estimadores DD 2x2 posibles que comparan grupos de tiempo entre sí. En particular, es un promedio ponderado de comparaciones entre: (1) unidades tratadas en algún momento del tiempo versus unidades nunca tratadas; (2) tratados "temprano" como grupo de tratamiento versus tratados "tarde" como grupo de control, antes de que comience el tratamiento de estos últimos (antes de $t_{l}^{*}$, los tratados "tarde" pueden actuar como control dado que todavía no empezó su tratamiento), y de manera análoga (3) tratados "tarde" como grupo de tratamiento versus tratados "temprano" como control, una vez que comienza el

\footnotetext{
${ }^{26} \mathrm{El}$ trabajo establece $t_{k}^{*}=\frac{34}{100} T$ y $t_{l}^{*}=\frac{85}{100} T$
} 
tratamiento de los tratados "tarde" (después de $t_{l}^{*}$ los tratados "temprano" pueden funcionar como control a pesar de haber sido tratados porque su estatus de tratamiento no cambia).

Formalmente:

$$
\hat{\beta}^{D D}=\sum_{k \neq U} s_{k U} \hat{\beta}_{k U}^{2 x 2}+\sum_{k \neq U} \sum_{l>k} s_{k l}\left[\mu_{k l} \hat{\beta}_{k l}^{2 x 2, k}+\left(1-\mu_{k l}\right) \hat{\beta}_{k l}^{2 x 2, l}\right]
$$

Donde $\widehat{\boldsymbol{\beta}}^{\boldsymbol{D} \boldsymbol{D}}$ es el estimador de diferencias en diferencias del modelo lineal con efectos fijos cuando el tratamiento es secuencial, $\widehat{\boldsymbol{\beta}}_{\boldsymbol{k} \boldsymbol{U}}^{2 x \boldsymbol{2}}, \widehat{\boldsymbol{\beta}}_{\boldsymbol{k} \boldsymbol{l}}^{2 x \boldsymbol{2}, \boldsymbol{k}}$ y $\widehat{\boldsymbol{\beta}}_{\boldsymbol{k} \boldsymbol{l}}^{2 x \boldsymbol{2}, \boldsymbol{l}}$ son las comparaciones (1), (2) y (3), respectivamente ${ }^{27}$, y $\boldsymbol{s}_{\boldsymbol{k} U}, \boldsymbol{s}_{\boldsymbol{k} \boldsymbol{l}}$ y $\boldsymbol{\mu}_{\boldsymbol{k} \boldsymbol{l}}$ son los pesos asignados a cada estimador $2 \mathrm{x} 2$, que dependen positivamente del tamaño de los grupos y de la varianza del tratamiento de cada uno de ellos. ${ }^{28}$ Adicionalmente, $\sum_{k \neq U} \boldsymbol{s}_{k U}+\sum_{k \neq U} \sum_{l>k} \boldsymbol{s}_{k l}=\mathbf{1}$.

Por último, define $K$ períodos distintos en los que se recibe tratamiento, de manera que con $K$ grupos más el "nunca tratado", el estimador $\hat{\beta}^{D D}$ proviene de $K^{2}$ estimadores DD 2x2 distintos: $K^{2}-K$ comparaciones solo de "tiempo" -las (2) y (3)- y $K$ comparaciones del grupo nunca tratado con cada uno de los $K$ grupos tratados.

\footnotetext{
${ }^{27}$ En el caso de la comparación (1), el autor menciona que con solo uno de los dos grupos de tratamiento, una estimación de DD del modelo lineal con efectos fijos, se reduce al caso que compara un solo grupo tratado con el grupo no tratado, por eso se indexa con $k$.

${ }^{28}$ La variación del tratamiento indica qué tan cerca del inicio/final del panel se activa el tratamiento. Es más alta para los grupos tratados en el medio del panel y más baja para los grupos tratados en los extremos.
} 